\title{
Directions for Open Access Publishing
}

\section{William J Teahan*}

School of Computer Science, Bangor University, Bangor, Wales, UK

\section{Introduction}

Recently, open access publishing has received bad press with labels such as "predatory publishing" becoming associated with it. In many cases, open access publishing as a whole has been unfairly labelled under the same predatory umbrella. The purpose of this editorial is not to convince the reader of the rightness or even ethics of publishing in journals for which other academics have raised strong concerns, but to try to convince the reader that open access publishing holds promise of a better model for scientific publishing in the future, and that exploration of improved methods of publishing should be actively explored and indeed encouraged. At the same time, where existing practices fall short of a professional standard, then this should be highlighted, and rather than being dismissive and responding only with at times destructive criticism, the academic community as a whole should work together to help make the improvements that are needed.

A cause for concern for open access publishing has been the continuing evidence of poor reviewing practices often employed by publishers who use the open access model. However, there is strong evidence that the problem of poor review is not just prevalent with the open access model; it is also endemic throughout all forms of academic publishing. Michael Eisen [1] states bluntly in his recent blog: “...the lesson [is] not that open access is bad, but that peer review is a joke”. He goes further: "There are deep problems with science publishing. But the way to fix this is not to curtain open access publishing. It is to fix peer review".

Continuing, he states: "First, and foremost, we need to get past the antiquated idea that the singular act of publication-or publication in a particular journal-should signal for all eternity that a paper is valid, let alone important. Even when people take peer review seriously, it is still just represents the views of 2 or 3 people at a fixed point in time". The list of problems that occur with the anonymous peer review process are many: wrong reviewers being selected; reviews done too quickly; reviews being just plain wrong; evidence that the article being reviewed has not been read and/or understood properly; articles being rejected but reappearing under the name of one of the reviewers later on; articles having good reviews but still being rejected by the editorial team; articles having two good reviews but one scathing one completely at odds with the other two reviews; one or more of the reviews being just a few sentences with little or no justification; reviews being unprofessionally condescending, rude or extremely critical rather than politely encouraging; and so on.

As Eisen points out, the peer review process needs to be more transparent and persist for the life of the published work. It is the discussion and debate that follows the submission and subsequent publication of an article that is one of the most important aspects of scientific publishing. Anonymous peer review does not allow part of this discussion and debate to be open for all to see or allow readers to follow up with their own comments concerning whether they agree or disagree with a review. A mechanism such as an online forum for readers to share their own views on the quality of some article is now expected for many online publications (for example, news websites and blogs), and the lack of this service will make publishers increasingly seem out of touch. This begs the question-why don't more academic publishers provide this service?

Open access publishing holds the promise of improved scientific publishing because online publishing opens up many new opportunities that previously were unavailable. Open access publishers should also embrace new methods of peer review that have been adopted or are being developed. Table 1 lists some of the different methods of 'open peer review' and 'open peer commentary' that are currently being adopted where part or all of the review process and subsequent discussion is made more transparent. Apart from the first method listed, the other methods address to some extent the accountability and non-transparency issues that arise with traditional anonymous peer review and can also help overcome problems of slow review, systemic bias and possible fraud (Table 1).

In addition, there are many cases where the contribution of an anonymous reviewer has had a significant impact on the quality of an article (as often mentioned in the acknowledgment section of a published article). In an open and transparent publication process, good quality reviews should be highly valued (and mentioned in $\mathrm{CVs}$ ). Paid review should also be considered as an option to encourage

\begin{tabular}{|l|l|}
\hline Method & Description \\
\hline Cross peer review & $\begin{array}{l}\text { Rather than review independently, reviewers are } \\
\text { encouraged to comment on each other's report [2]. } \\
\text { Example: Nature's The EMBO Journal. }\end{array}$ \\
\hline Interactive peer review & $\begin{array}{l}\text { The interactive peer review follows an initial independent } \\
\text { peer review. Collaboration between authors and } \\
\text { reviewers is conducted through an on-line forum until a } \\
\text { consensus is reached [2]. } \\
\text { Example: Frontiers. }\end{array}$ \\
\hline $\begin{array}{l}\text { Post publication peer } \\
\text { review }\end{array}$ & $\begin{array}{l}\text { Speedy publication is ensured by rejecting only articles } \\
\text { that are clearly inappropriate. Published articles are } \\
\text { initially labelled as "Awaiting peer review" until they pass } \\
\text { the peer review process. Review reports are available for } \\
\text { everyone to view alongside the article [2]. } \\
\text { Examples: F1000Research; WebmedCentral. }\end{array}$ \\
\hline Open peer commentary & $\begin{array}{l}\text { Non-anonymous commentary on a peer-reviewed article } \\
\text { is solicited from experts post publication and this is } \\
\text { published with the author's response. } \\
\text { Example: Current Anthropology. }\end{array}$ \\
\hline $\begin{array}{l}\text { Author-guided peer } \\
\text { review } \\
\text { their work. Reviews are available alongside the article, and } \\
\text { direct communication between authors and reviewers is } \\
\text { encouraged throughout the review process [3]. } \\
\text { Example: LIBRE. }\end{array}$ \\
\hline
\end{tabular}

Table 1: Some methods for open peer review and open peer commentary.

*Corresponding author: William Teahan, School of Computer Science, Bangor University, Bangor, Wales, UK, Tel: 01248382686; E-mail: w.j.teahan@bangor.ac.uk

Received August 01, 2014; Accepted August 05, 2014; Published August 07, 2014

Citation: Teahan WJ (2014) Directions for Open Access Publishing. J Comput Sci Syst Biol 7: e110. doi:10.4172/jcsb.1000e110

Copyright: @ 2014 Teahan WJ. This is an open-access article distributed under the terms of the Creative Commons Attribution License, which permits unrestricted use, distribution, and reproduction in any medium, provided the original author and source are credited. 
experts with busy schedules to contribute to the discussion.

As stated, open access publishing as well as traditional subscription style publishing should be seen more as a forum for discussion rather than as a vehicle for personally increasing the number of publications (rather than the 'publish or perish' mantra, a 'publish for discussion' mantra should be adopted). Open access publishing can help facilitate this discussion amongst as wide an audience as possible by using a concerted effort to disseminate publications across the Internet, for example, by using effective web design and search engine optimisation techniques.

Measures for the quality of an article should be related to the number of its citations rather than the perceived quality of a particular journal. Sites such as Web of Science do not index all journals with coverage in some areas being less complete than in others [4]. Importantly, they do not index local university repositories, or articles published as preprints on websites, or informally published written material called 'grey literature'. In contrast, sites that search and index the entire Internet, such as Google Scholar, provide a more meaningful measure of the impact that an article has in today's publishing landscape.

Of course, traditional subscription publishers also have the opportunity to publish articles on the web in conjunction with the printed publication. Increasingly, this will result in the differences between open access publishing and subscription publication becoming blurred, with both offering the same quality of services such as effective search engine optimisation. The only difference will be that one form of publication will be open to all; the other will require some fee to be paid before access is granted. The later model clearly goes against the ethos under which much of the Internet was founded and will inevitably result in a reduced level of dissemination across the Internet as a result (since many potential readers of the article will be unwilling to pay to access publications that they are unsure will be of interest to them).

Another form of open access publishing involves the use of advertising to offset the cost of publication and generate ongoing revenues for both the publisher and author. Rather than the authors paying a fee for publication, the article or manuscript is made available for free after peer review, but with advertisements placed on the website where the article is viewed or downloaded (perhaps using Google Adsense), or inserted directly into the manuscript by the editors prior to publication. This latter form of open access publishing is most effective for larger manuscripts (for example, for textbooks and monographs) whereas there has been comparatively little uptake of advertising amongst open access journals [5] perhaps due to a lack of understanding of what forms of advertising are most effective.

In a survey of 377 open access journals, Frantsvåg [5] provides the following reasons for why publishers have not adopted advertising to potentially increase their revenue and offset publications costs for both the publisher and the author: they have a policy not to have advertising (43\%); they believe that scientific journals should not have advertising (24\%); they believe that the potential income is too small (14\%); they do not want the extra work (17\%); their publishing solution does not easily allow advertising (14\%); they haven't thought of it (26\%); and other (14\%). From several of the responses to this survey, it is clear that open access publishers should review their advertising policy considering that online advertising can be implemented using Google Adsense with very little effort. This future direction of open access publishing is an important one, especially if this can reduce the increasing cost of open access publishing for authors, with the potential to provide further non-trivial revenue streams for well-received articles.
One publisher that uses the 'in-book ads' model when publishing online text books and monographs is the Danish publisher bookboon. The author of this article has published four e-books in a series on Artificial Intelligence [6] with bookboon in 2010. Over the last two years, there have been over 600,000 downloads of these books demonstrating the popularity of the ad-based open access method of publishing. This compares with numbers as low as 150 to 300 often claimed is the usual number of book sales for a complex monograph printed in the traditional manner [7].

Another important direction for open-access publishing is the use of supplementary material (videos, blogs, animations, web-based programs) alongside the open-access publication in order to enhance the quality of the publication. A unique feature of the Artificial Intelligence e-books described above is the use of the agent-oriented language NetLogo to provide code to demonstrate the algorithms and techniques that are discussed in the books. There are 63 NetLogo programs (called 'models') that can be run directly or downloaded from the files.bookboon.com/ai website. A screenshot from one of the models-the Follow Trail model-is shown in Figure 1. The model can be used to explore the effectiveness of code generated by various evolutionary algorithms for the artificial ant problem (an example of which is shown in the 'Ant-Actions' box on the left of the figure). The model demonstrates what happens when this code is executed by a colony of artificial ants following a food trail. The food trail in this case is a brown-coloured ring shown in the environment on the right of the figure and the artificial ants are multi-coloured for visualisation purposes.

Two of the four books in the e-book series are textbooks that provide an introduction to the subject of Artificial Intelligence. The remaining two books provide exercises as well as discuss how the NetLogo models can be used to provide further insights into the theory. The idea of the models is to provide visualisations and animations of the execution of the various algorithms and techniques. They also provide an opportunity for performing modelling and simulation in NetLogo's 2D virtual environment by making it very easy to explore different model settings through the use of each model's interface sliders and choosers.

There are several features of NetLogo that make it an ideal means for exploring simulation and modelling related to the areas of computer science and systems biology (and therefore as a means for enhancing the quality of open access publications with supplementary material). NetLogo code is very readable (similar to pseudo-code) and compact and therefore it is useful for providing working code examples for

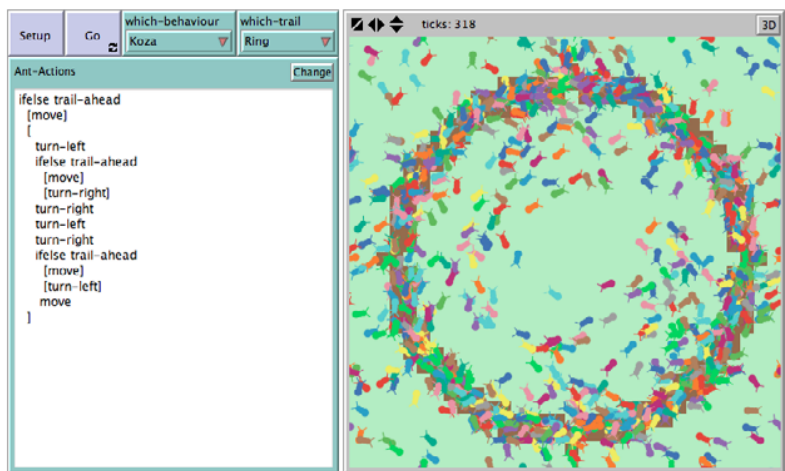

Figure 1: Screenshot of the Follow Trail NetLogo model used in conjunction with the Artificial Intelligence e-book series [6] at files.bookboon.com/ai. 
pedagogical purposes. As a rapid prototyping language, it is an ideal platform for experimentation. It is also easy to create QuickTime movies from the simulation, or create applets to run the simulation in a web browser, or use the Behaviour Space tool to run many simulations with different parameters and export generated data to a spreadsheet which then can be later analysed using other tools (such as data mining software).

\section{Conclusion}

This article has explored some of the current issues related to open-access publishing. Its purpose has been to encourage future publishers and authors of open access publications to explore further the new frontiers that have been opened up by the adoption of the open access model. It has suggested some directions with which these frontiers may be explored. A fundamentally important direction will be the exploration of methods to enhance the quality of the peer review of the publications. This article has provided some examples such as open peer review, open peer commentary and author-guided peer review. Publishers have been encouraged to consider the adoption of advertising as this has the potential for reducing the increasing costs to authors of open access publishing. Another direction that requires further exploration is the use of supplementary materials to add value to open access publications. One example was described of the use of web-based applications implemented in NetLogo to provide more effective demonstrations of the ideas and concepts explored in the publications by using additional visualisations, animations, and modelling and simulation techniques.

\section{References}

1. Eisen M (2013) "it is NOT junk".

2. Adam G (2014) The Future of Publishing: New models of Peer Review.

3. Pandelis Perakakis (2014) New forms of open peer review will allow academics to separate scholarly evaluation from academic journals. The London School of Economics and Political Science.

4. (2014) Web of Science Wikipedia entry.

5. Jan Eric Frantsvåg (2010) The role of advertising in financing open access journals. First Monday 15:1-3.

6. William Teahan (2010) Artificial Intelligence e-book series.

7. Katherine R (2010) Bound for Glory. 